\title{
Relationship between 3D Power Doppler Ultrasound and Serum MMP-2 and Ang-2 Levels in Pregnant Women with Preeclampsia
}

\author{
Xiaoyu Lin, Xiuping Zhou, Huipei Jin and Yaping Zhao \\ Department of Ultrasound, The Second Affiliated Hospital and Yuying Children's Hospital of Wenzhou Medical University, China
}

\begin{abstract}
Objective: To explore the relationship between 3D power Doppler ultrasound (3D-PDU) and serum MMP-2 and Ang-2 levels in pregnant women with preeclampsia (PE).

Study Design: Comparative analytical study.

Place and Duration of Study: The Second Affiliated Hospital and Yuying Children's Hospital of Wenzhou Medical University, China, from October 2017 to January 2020.

Methodology: One hundred and thirty-four pregnant women with PE were selected as the research subjects, with 67 cases in mild group and 67 cases in severe group. Sixty-seven healthy primigravidas were selected as normal control group. Serum MMP-2 and Ang-2 were detected. All subjects underwent prenatal 3D-PDU examination, and VI, FI and VFI were measured.

Results: Compared to normal control group, serum MMP-2 and Ang-2 levels of mild group and severe group decreased successively (all $p<0.001$ ); $\mathrm{VI}, \mathrm{Fl}$, and VFI of mild group and severe group decreased successively (both $p<0.001$ ). Pearson correlation analysis showed that serum MMP-2 in pregnant women with PE was positively correlated with VI, FI, VFI $(r=0.940, p<0.001, r$ $=0.938, p<0.001$, and $r=0.921, p<0.001$, respectively); and serum Ang-2 was positively correlated with VI, FI, VFI $(r=0.958$, $p<0.001 ; r=0.960 ; p<0.001 ; r=0.945, p<0.001$, respectively).

Conclusion: 3D-PDU has a certain application value in the diagnosis of pregnant women with PE, variation tendency of VI, FI and VFI detected by 3D-PDU is closely associated with serum MMP-2 and Ang-2 levels.
\end{abstract}

Key Words: Preeclampsia, Pregnant women, MMP-2, Ang-2, 3D power Doppler ultrasound (3D-PDU).

How to cite this article: Lin X, Zhou X, Jin H, Zhao Y. Relationship between 3D Power Doppler Ultrasound and Serum MMP-2 and Ang-2 Levels in Pregnant Women with Preeclampsia. J Coll Physicians Surg Pak 2020; 30(08):810-814.

\section{INTRODUCTION}

Preeclampsia (PE), a pregnancy-specific disorder, remains a leading cause of maternal and perinatal morbidity and mortality. ${ }^{1}$ The specific pathogenesis of PE still remains unclear. ${ }^{2}$ Placenta may play a major role in its pathogenesis. ${ }^{3}$ The pathological manifestations of placenta in patients with PE are shallow placental implantation, small villi area, and poorly developed blood vessels in the villi. ${ }^{4}$ The degradation of extracellular matrix (ECM) is a key part of trophoblast invasion. Matrix metalloproteinase-2 (MMP-2) plays a pivotal role in cell invasion. Its substrate is type IV collagen, the main component of ECM. MMPs are closely linked to trophoblastic invasion and infiltration abilities. ${ }^{5}$ Serum MMP-2 expression is increased in patients with PE, indicating that MMP-2 may bea major risk factorin pregnancy-induced hypertension. ${ }^{6}$

Correspondence to: Yaping Zhao, Department of Ultrasound, The Second Affiliated Hospital and Yuying Children's Hospital of Wenzhou Medical University, China E-mail: qtii2t@163.com

Received: May 10, 2020; Revised: August 06, 2020; Accepted: August 12, 2020

DOI: https://doi.org/10.29271/jcpsp.2020.08.810
Ang-2, a member of the Angs family, is involved in physiological and pathological angiogenesis. ${ }^{7,8}$ It is an essential factor in the development of placenta. Low level of Ang-2 may reduce the invasion ability of trophoblast cells, resulting in poor formation of placental vascular network. Next, continuous hypoxia of placental tissues produces a large amount of cytotoxic substances into blood, and then PE is induced. Studies have shown that the Ang-1/Ang- 2 ratio in plasma constitutes a possible biomarker for prediction of PE. ${ }^{9,10}$

Three-dimensional power Doppler ultrasound (3D-PDU) is a technique that forms images using the density, scattering intensity or energy distribution of red blood cells in the bloodstream, namely the number of red blood cells per unit area and the amplitude of signals. 3D-PDU, not limited by angle-dependent aliasing and low sensitivity to low-velocity blood flow, has a wide range of display signals. It shows strengths in observing the imaging of small vessels, low-velocity blood flow and its vascular space, and is therefore suitable for monitoring the placental vascular tree. After image collection, vascularization index (VI), flow index (FI) and vascularization flow index (VFI) can be calculated by 3D reconstruction technology and VOCAL software. ${ }^{11}$ 
Table I: Variation of serum MMP-2 and Ang-2 levels.

\begin{tabular}{|l|c|c|c|c|}
\hline Parameter & Normal control group $(\mathbf{n = 6 7 )}$ & Mild group $(\mathbf{n = 6 7 )}$ & Severe group (n=67) & p-value \\
\hline MMP-2 $(\mu \mathrm{g} / \mathrm{L})$ & $2.27 \pm 0.10$ & $1.46 \pm 0.07$ & $0.59 \pm 0.06$ & $<0.001$ \\
\hline Ang-2 $(\mu \mathrm{g} / \mathrm{L})$ & $2.18 \pm 0.22$ & $1.37 \pm 0.13$ & $0.49 \pm 0.07$ & $<0.001$ \\
\hline
\end{tabular}

Table II: Comparison of VI, FI and VFI of placental bed vessels in each group.

\begin{tabular}{|c|c|c|c|c|}
\hline Parameter & Normal control group $(n=67)$ & Mild group $(n=67)$ & Severe group $(n=67)$ & p-value \\
\hline $\mathrm{VI}$ & $20.85 \pm 2.25$ & $14.68 \pm 1.35$ & $8.38 \pm 0.81$ & $<0.001$ \\
\hline $\mathrm{FI}$ & $43.97 \pm 4.75$ & $39.29 \pm 4.22$ & $21.30 \pm 2.29$ & $<0.001$ \\
\hline VFI & $11.11 \pm 1.20$ & $8.95 \pm 0.97$ & $5.32 \pm 0.57$ & $<0.001$ \\
\hline
\end{tabular}

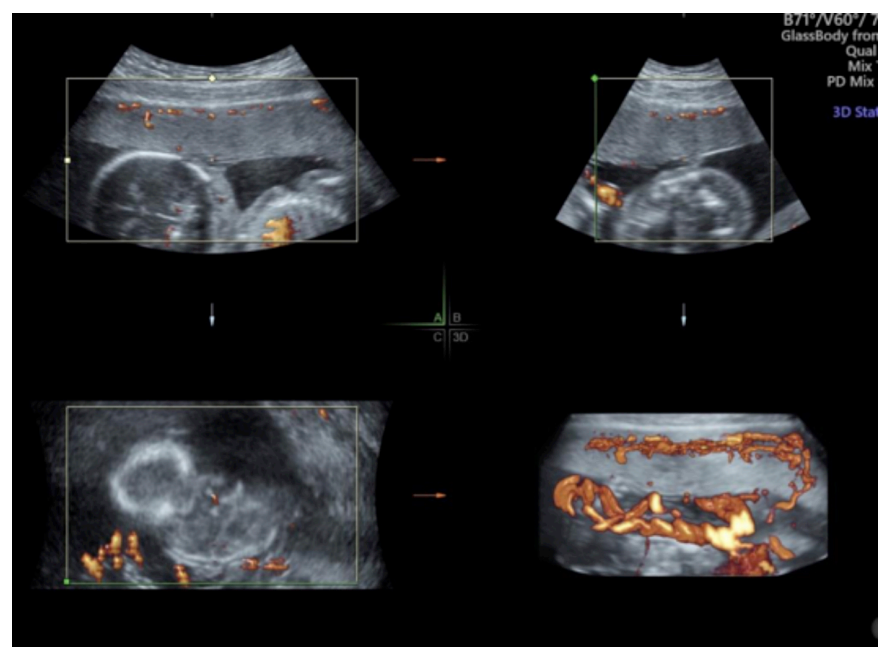

Figure 1 (A): 3D power imaging of the placenta in pregnant women with PE.

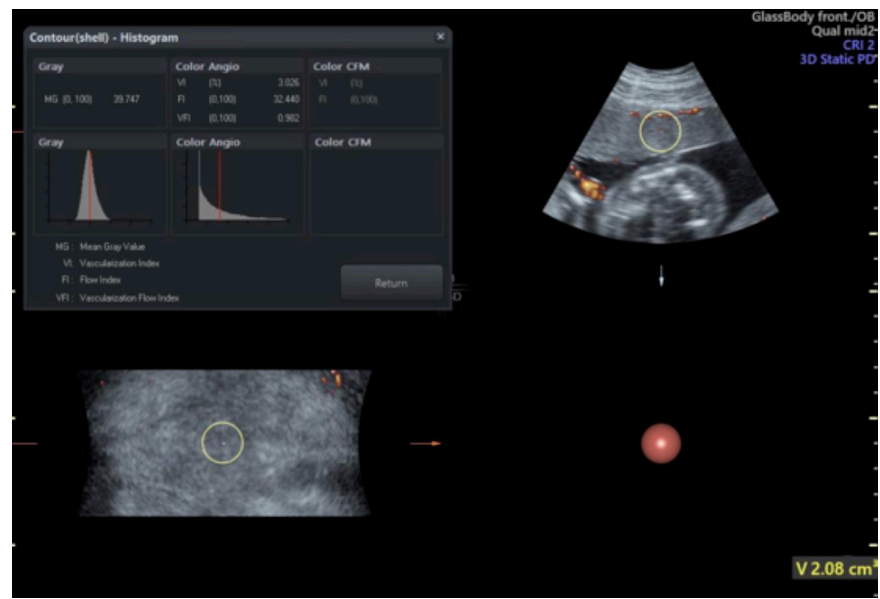

Figure 1 (B): 3D power imaging index of the placenta in pregnant women with PE.

Therefore, 3D-PDU can show the density degree of blood vessels in the tissue, and the 3D spatial relationship of blood vessel shapes and branches. ${ }^{12}$ At present, there are few reports on the correlation between 3D-PDU and serum MMP-2 and Ang-2 in pregnant women with $\mathrm{PE}$.

The purpose of this study was to explore the relationship between 3D-PDU and serum MMP-2 and Ang-2 levels in pregnant women with PE.

\section{METHODOLOGY}

This comparative analytical study was conducted at The
Second Affiliated Hospital and Yuying Children's Hospital of Wenzhou Medical University, China from October 2017 to January 2020. The study was approved by the Ethics Committee of the Hospital. The 134 pregnant women with PE were selected as the research subjects, with 67 cases in the mild group and 67 cases in the severe group. The diagnostic criteria for mild PE were blood pressure $\geq 140 / 90 \mathrm{mmHg}$ after 20 weeks of gestation; and urine protein $\geq 0.3 \mathrm{~g} / 24$ hour or random urine protein $(+)$, which may be accompanied by upper abdominal discomfort or headache. The diagnostic criteria for severe PE were those with hypertension, proteinuria and at least one of the clinical symptoms or signs after 20 weeks of gestation, including systolic blood pressure $\geq 160-180 \mathrm{mmHg}$ or diastolic blood pressure $\geq 110$ $\mathrm{mmHg} ; 24 \mathrm{~h}$ urinary protein $>2.0 \mathrm{~g}$ or random urinary protein $(+++)$ or more; central nervous system dysfunction, mental state change and severe headache (frequent, not relieved by regular analgesics), cerebrovascular accident, blurred vision, spotted hemorrhage in the fundus, cortical blindness in very few cases; hepatocyte dysfunction, hepatocyte injury, serum transaminases increased by at least 2 times; hepatic capsule swelling symptoms, such as upper abdominal or upper right quadrant pain, subcapsular hemorrhage or liver rupture; oliguria, 24 hour urine volume $<500$ $\mathrm{mL}$; pulmonary edema, heart failure; platelet count $<100 \times 10^{9} / \mathrm{L}$, coagulopathy; microangiopathic hemolysis, elevated blood lactate dehydrogenase; and / or fetal growth restriction, oligohydramnios, and placental abruption.

Inclusion criteria were primiparas with singleton pregnancy, no high-risk scores; accurate check of gestational week, no history of fetal protection in early pregnancy; no medical or surgical diseases; pre-pregnancy standard body mass index $18.5-28 \mathrm{~kg} / \mathrm{m}^{2}$; basic information provided during follow-up; and those willing to be followed up until being discharged after delivery. Exclusion criteria were lack of regular prenatal examination lost to follow-up during the research; delivered elsewhere, complicated with intrahepatic cholestasis in the middle and late pregnancy; and those with heart, liver, lung, kidney and other vital organ diseases, and benign tumors (such as ovarian cysts and uterine fibroids) during pregnancy. Meanwhile, 67 healthy primigravidas who gave birth in our hospital during the same period were selected as the normal control group.

Five $\mathrm{mL}$ peripheral blood was collected from all the subjects 
after admission, and the centrifuged serum samples were taken to detect serum MMP-2 and Ang-2 levels of the subjects using enzyme-linked immunosorbent assay. All subjects underwent prenatal 3D-PDU examination (probe frequency 5-8 MHz), and VI, FI and VFI were measured using $3 \mathrm{D}$ reconstruction technology and VOCAL software. The 3DPDU examination was measured by the same skilled physician.

SPSS version 25.0 statistical software was used for analysis. Kolmogorov-Smirnov test was used to evaluation of the normality of quantitative data. Measurement data with normal distribution were expressed by mean \pm SD, single factor analysis of variances with $L S D$-t test was used for comparison among groups. Pearson correlation test was used for correlation analysis. The $p<0.05$ was statistically significant.

\section{RESULTS}

In the mild group, patients aged 25-38 (30.52 \pm 2.59 ) years, with 33-42 (38.15 \pm 3.37 ) weeks of gestation and body mass index of $20-27(24.49 \pm 2.38) \mathrm{Kg} / \mathrm{m}^{2}$ before pregnancy. In the severe group, patients aged 26-39 (30.94 \pm 2.83$)$ years, with 33-42 (38.34 \pm 3.62 ) weeks of gestation and body mass index of $21-28(24.67 \pm 2.45) \mathrm{Kg} / \mathrm{m}^{2}$ before pregnancy. In the normal control group, patients were aged 24-38 (30.31 \pm 2.47 ) years, with 34-41 (37.97 \pm 2.55 ) weeks of gestation and body mass index of 20-28 (24.56 \pm 2.62$) \mathrm{Kg} / \mathrm{m}^{2}$ before pregnancy. Compared to the normal control group, serum MMP-2 and Ang-2 levels of the mild group and severe group decreased successively, and the differences were statistically significant (all $p<0.001$, Table I).

The 3D power imaging of the placenta in pregnant women with PE was shown in Figure $1 A$, and the $3 D$ power imaging index was shown in Figure 1B. Compared to the normal control group, $\mathrm{VI}, \mathrm{FI}$, and VFI of the mild group and severe group decreased successively, and the differences were statistically significant (both $p<0.001$, Table II).

Pearson correlation analysis of serum MMP-2, Ang-2 and VI, FI, VFI were conducted in 134 pregnant women with PE. The results showed that serum MMP-2 in pregnant women with PE was positively correlated with VI, FI, VFI ( $r=0.940, p$ $<0.001, r=0.938, p<0.001, r=0.921, p<0.001$, respectively); and serum Ang-2 was positively correlated with VI, FI, VFI $(r=0.958, p<0.001 ; r=0.960 ; p<0.001 ; r=$ $0.945, p<0.001$, respectively.

\section{DISCUSSION}

3D-PDU technology is simple, safe and non-invasive with no need for contrast and exposure to radiation. It provides a new method for studying the overall blood flow of a certain tissue or organ, and can qualitatively assess the blood vessel type and distribution characteristics. Once the researcher has determined an area of interest and collected the images, the three blood flow parameters can be calculated through 3D reconstruction technology and VOCAL software, namely VI represents the number of blood vessels in a unit volume in the area of interest, $\mathrm{Fl}$ the average strength of the blood flow signal in the target volume, and VFI the comprehensive expression of the first two parameters. Therefore, 3D-PDU can not only show the density degree of blood vessels in the tissue and the spatial relationship of blood vessel shapes and branches, but also provide indirect data on the number of blood cells per unit volume.

A study have shown that $\mathrm{VI}, \mathrm{FI}$, and VFI are affected by external factors, such as blood flow, attenuation, blood vessel number, and red blood cell density. ${ }^{13} 3 \mathrm{D}-\mathrm{PDU}$ has a better imaging effect on blood flow parameters, such as VI, $\mathrm{FI}$ and VFI compared to the 2D technology. ${ }^{14}$ Mihu et al. used quantitative 3D power Doppler to measure blood flow parameters VI, FI, VFI in patients with pregnancy-induced hypertension, and suggested that blood flow parameters could sensitively show changes in placental blood perfusion in patients with pregnancy-induced hypertension. ${ }^{15}$

The results of this study showed that compared to the normal control group, VI, FI, and VFI decreased successively in the mild group and severe group, which is basically similar to what has been reported by Neto et al. ${ }^{11}$ This indicates that the placental blood perfusion of pregnant women with PE is reduced, and placental ischemia is present. The reason for the significant decrease in $\mathrm{VI}, \mathrm{VFI}$, and $\mathrm{FI}$ values of pregnant women with PE may be associated with the decrease in the number of blood vessels per unit volume in the placenta and the decrease in blood flow amplitude.

When the expression of MMP-2 in the placenta tissue is insufficient, the placental trophoblast cells lack sufficient power to penetrate the decidua of the uterus, and this is the important theoretical basis for the study on the occurrence of shallow placental implantation. ${ }^{16}$ Isaka et al. revealed that invasive ability of trophoblasts might be regulated by MMP-2. ${ }^{17}$ The results of this study showed that compared to the normal control group, serum MMP-2 levels in patients with PE were significantly reduced, which is similar to what has been reported by Narumiya et al. It also revealed that serum MMP-2 levels were further reduced with the aggravation of PE, and the difference between the severe group and the mild group was statistically significant. ${ }^{18}$ This indicates that the invasiveness of placental trophoblast cells in patients with PE is significantly reduced, which is closely associated with the severity of the disease.

The expression levels of Ang-2 mRNA and protein in the placenta tissue of pregnant women with PE are reduced, resulting in a decrease in serum Ang-2 levels; and Ang-2 may be involved in the pathogenesis of PE by affecting the placenta formation in early pregnancy. ${ }^{19}$ The results of this study showed that compared to the normal control group, serum Ang-2 levels in PE patients are significantly reduced, 
which is similar to what has been reported by Hirokoshi et al. ${ }^{20}$ It also revealed that serum Ang-2 levels were further reduced with the aggravation of $\mathrm{PE}$, and the difference between the severe group and the mild group was statistically significant. It shows that the determination of serum Ang-2 concentration in pregnant women can be used as a useful indicator for diagnosing and predicting PE.

Zhang et al. confirmed trends in placenta and indexes (VI, FI and VFI) of 3D-PDU detection of MMP-2 expression was consistent. ${ }^{21}$ In this study, Pearson correlation analysis showed that serum MMP-2 and Ang-2 in pregnant women with PE are both positively correlated with VI, FI and VFI. It suggests that parameters VI, FI and VFI measured by the 3DPDU technology can reflect the placental blood perfusion of pregnant women with $\mathrm{PE}$, and they, together with joint detection of serum MMP-2 and Ang-2, have certain clinical significance for monitoring risk and obtaining knowledge of the placental function in pregnant women with PE.

\section{CONCLUSION}

3D-PDU has a certain application value in the diagnosis of pregnant women with PE, variation tendency of VI, FI and VFI detected by 3D-PDU is closely associated with serum MMP-2 and Ang-2 levels.

\section{ACKNOWLEDGEMENT:}

The research was funded by the Public Welfare Science and Technology Planning Projects of Wenzhou Science and Technology Bureau (No.Y20170830).

\section{ETHICAL APPROVAL:}

This study was conducted with the approval from the Ethics Committee of The Second Affiliated Hospital and Yuying Children's Hospital of Wenzhou Medical University, China.

\section{PATIENTS' CONSENT:}

All patients signed a document of informed consent.

\section{CONFLICT OF INTEREST:}

Authors declared no conflict of interest.

\section{AUTHORS' CONTRIBUTION:}

XL: Acquired data; drafted manuscript; contributed substantially in revision.

$X Z, H J$ : Acquired data; drafted manuscript.

YZ: Drafted manuscript; read and approved the final manuscript.

\section{REFERENCES}

1. Ali Z, Khaliq S, Zaki S, Ahmad HU, Lone KP. Differential expression of placental growth factor, transforming growth factor- $\beta$ and soluble endoglin in peripheral mononuclear cells in preeclampsia. J Coll Physicians Surg Pak 2019; 29(3):235-9. doi: 10.29271/jcpsp.2019.03.235.

2. Hosapatna Basavarajappa D, Saha PK, Bagga R, Khandelwal $\mathrm{N}$, Modi M. Neuroradiological perspectives of severe preeclampsia and eclampsia spectrum - Correlation from poste- rior reversible encephalopathy syndrome. Pregnancy Hypertens 2020; 20:119-23. doi: 10.1016/j. preghy. 2020.04.003.

3. Iriyama T, Wang W, Parchim NF, Sayama S, Kumasawa K, Nagamatsu $T$, et al. Reciprocal upregulation of hypoxia-inducible factor- $1 \alpha$ and persistently enhanced placental adenosine signaling contribute to the pathogenesis of preeclampsia. FASEB J 2020; 34(3):4041-54. doi: 10.1096/fj. 201902583R.

4. Stanek J. Histological features of shallow placental implantation unify early-onset and late-onset preeclampsia. Pediatr Dev Pathol 2019; 22(2):112-22. doi: 10.1177/ 109352 6618803759.

5. Rahat B, Sharma R, Bagga R, Hamid A, Kaur J. Imbalance between matrix metalloproteinases and their tissue inhibitors in preeclampsia and gestational trophoblastic diseases. Reproduction 2016; 152(1):11-22. doi: 10.1530/ REP16-0060.

6. Laskowska M. Altered maternal serum matrix metalloproteinases MMP-2, MMP-3, MMP-9, and MMP-13 in severe earlyand late-onset preeclampsia. Biomed Res Int 2017; 2017:6432426. doi: 10.1155/2017/6432426.

7. Kappou D, Sifakis S, Konstantinidou A, Papantoniou N, Spandidos DA. Role of the angiopoietin/Tie system in pregnancy (Review). Exp Ther Med 2015; 9(4):1091-6. doi: 10.3892/etm.2015.2280.

8. Lefere S, Van de Velde F, Hoorens A, Raevens S, Van Campenhout S, Vandierendonck A, et al. Angiopoietin-2 promotes pathological angiogenesis and is a therapeutic target in murine nonalcoholic fatty liver disease. Hepatology 2019; 69(3):1087-1104. doi: 10.1002/hep. 30294.

9. Machado JSR, Machado MSR, Bertagnolli TV, Martins LAB, Freitas SF, Ovidio PP, et al. Role of plasma PIGF, PDGF-AA, ANG-1, ANG-2, and the ANG-1/ANG-2 ratio as predictors of preeclampsia in a cohort of pregnant women. Pregnancy Hypertens 2019; 16:105-11. doi: 10.1016/j.preghy.2019. 03.011.

10. Bolin M, Wiberg-Itzel E, Wikström AK, Goop M, Larsson $A$, Olovsson $M$, et al. Angiopoietin-1/angiopoietin-2 ratio for prediction of preeclampsia. Am J Hypertens 2009; 22(8): 891-5. doi: 10.1038/ajh.2009.97.

11. Neto RM, Ramos JG. 3D power doppler ultrasound in early diagnosis of preeclampsia. Pregnancy Hypertens 2016; 6(1):10-6. doi: 10.1016/j.preghy.2015.11.003.

12. Cansu A, Kupeli A, Kul S, Eyuboglu I, Oguz S, Ozturk $\mathrm{MH}$, et al. Evaluation of the relationship between renal function and renal volume-vascular indices using 3D power doppler ultrasound. Eur J Radiol 2014; 83(7):1080-5. doi: 10.1016/j.ejrad.2014.04.007.

13. Raine-Fenning NJ, Nordin NM, Ramnarine KV, Campbell BK, Clewes JS, Perkins A, et al. Determining the relationship between three-dimensional power Doppler data and true blood flow characteristics: An in-vitro flow phantom experiment. Ultrasound Obstet Gynecol 2008; 32(4): 540-50. doi: 10.1002/uog.6110.

14. Pan HA, Wu MH, Cheng YC, Li CH, Chang FM. Quantification of doppler signal in polycystic ovary syndrome 
using three-dimensional power doppler ultrasonography: A possible new marker for diagnosis. Hum Reprod 2002; 17(1):201-6. doi: 10.1093/humrep/17.1.201

15. Mihu CM, Drugan T, Mihu D. Contribution of 3D power Doppler ultrasound to the evaluation of placental circulation in normal pregnancies and pregnancies complicated by preeclampsia. J Perinat Med 2012; 40(4):359-64. doi: 10.1515/jpm-2011-0105.

16. Vincent $Z \mathrm{~L}$, Mitchell MD, Ponnampalam AP. Regulation of MT1-MMP/MMP-2/TIMP-2 axis in human placenta. J Inflamm Res 2015; 8:193-200. doi: 10.2147/JIR.S88039.

17. Isaka $K$, Usuda $S$, Ito H, Sagawa $Y$, Nakamura H, Nishi H, et al. Expression and activity of matrix metalloproteinase 2 and 9 in human trophoblasts. Placenta 2003; 24(1):53-64. doi: 10.1053/plac.2002.0867.
18. Narumiya H, Zhang Y, Fernandez-Patron C, Guilbert LJ, Davidge ST. Matrix metalloproteinase-2 is elevated in the plasma of women with preeclampsia. Hypertens Pregnancy 2001; 20(2):185-94. doi: 10.1081/PRG-100106968.

19. Wang LJ, Chen WP, Peng W, Xu L, Sui AH, Ye YH. Correlation of angiopoietin-2 and angiopoietin-2 receptor expressions in serum and placenta with preeclampsia. Chin J Obstet Gynecol 2011; 46(3):167-71.

20. Hirokoshi K, Maeshima Y, Kobayashi K, Matsuura E, Sugiyama $H$, Yamasaki $Y$, et al. Increase of serum angiopoietin-2 during pregnancy is suppressed in women with preeclampsia. Am J Hypertens 2005; 18(9 Pt 1):1181-8. doi: 10.1016/j.amjhyper.2005.03.745.

21. Zhang YH, Li DM, Cai BY, Lin HY. The relationship between MMP-2 and TIMP-2 in postpartum placenta and 3D power Doppler ultrasound in preeclampsia. Maternal and child health care of China 2015; 30:3146-8. 